\title{
Estratégia Saúde da Família: gerenciamento do cuidado de enfermagem
}

\author{
Veridiana Fermino ${ }^{1}$, Simone Coelho Amestoy ${ }^{2}$, Bianca Pozza dos Santos ${ }^{3}$, Sidnéia Tessmer Casarin ${ }^{4}$
}

\footnotetext{
${ }^{1}$ Enfermeira. Pelotas, RS, Brasil. E-mail: verifermino@yahoo.com.br.

${ }^{2}$ Enfermeira, Doutora em Enfermagem. Professor Adjunto da Universidade Federal da Bahia. Salvador, BA, Brasil. E-mail: simoneamestoy@hotmail.com.

${ }^{3}$ Enfermeira, Mestre em Enfermagem. Discente do Programa de Pós-Graduação em Enfermagem, nível Doutorado, da Universidade Federal de Pelotas. Enfermeira da Prefeitura Municipal de Pelotas. Pelotas, RS, Brasil. E-mail: bi.santos@bol.com.br.

${ }^{4}$ Enfermeira, Mestre em Enfermagem. Discente do Programa de Pós-Graduação em Enfermagem, nível Doutorado, da Universidade Federal de Pelotas. Professor Assistente da Universidade Federal de Pelotas. Pelotas, RS, Brasil. E-mail: stcasarin@gmail.com.
}

Recebido: 18/08/2016.

Aceito: 08/12/2016.

Publicado: 18/04/2017.

\section{Como citar esse artigo:}

Fermino V, Amestoy SC, Santos BP, Casarin ST. Estratégia Saúde da Família: gerenciamento do cuidado de enfermagem. Rev. Eletr. Enf. [Internet]. 2017 [acesso em:_____ ] 19:a05. Disponível em: http://dx.doi.org/10.5216/ree.v19.42691.

\section{RESUMO}

Objetivou-se conhecer o entendimento dos enfermeiros atuantes na Estratégia de Saúde da Família acerca do conceito, das dificuldades e as estratégias utilizadas para desenvolver o gerenciamento do cuidado. Pesquisa qualitativa, descritiva e exploratória, realizada em Pelotas/RS/Brasil, participando 20 enfermeiros. A coleta de dados foi com entrevistas semiestruturadas, sendo analisadas conforme Análise Temática. Os enfermeiros entendem o gerenciamento do cuidado como a articulação entre gerenciamento e assistência. Quanto às dificuldades, há falta de estrutura física e de materiais, resistência ao lidar com colegas de trabalho, falta de autonomia e cargo de gerente distrital atribuído à pessoa sem conhecimentos na área da saúde. As estratégias sugeridas foram aproximação da gestão municipal com o serviço de saúde, investimentos em educação permanente e aumento do número de recursos humanos. Os enfermeiros entendem que a assistência e o gerenciamento estão interligados a fim de garantir o cuidado à população de seus territórios.

Descritores: Enfermagem; Estratégia Saúde da Família; Cuidados de Enfermagem; Administração de Serviços de Saúde.

\section{INTRODUÇÃO}

O gerenciamento do cuidado é entendido como uma atribuição do enfermeiro diretamente relacionada à busca pela qualidade assistencial e de melhores condições de trabalho para os profissionais ${ }^{(1)}$, em uma perspectiva que articule gerência e assistência, tendo como foco, o usuário do serviço de saúde e o cuidado em uma abordagem que supere o tecnicismo em direção à integralidade da atenção ${ }^{(2)}$.

Em sua origem teórica abrange uma relação dialética entre o saber fazer gerenciar e o saber-fazer 
cuidar. Para tanto, o saber-fazer da gerência do cuidado de enfermagem ancora-se no conhecimento científico, ético, estético e pessoal, face à complexidade do homem no que tange sua singularidade, multiplicidade e individualidade, bem como sua relação e inserção nos diversos cenários cotidianos ${ }^{(3)}$.

O gerenciamento do cuidado é exercido primordialmente pelo enfermeiro, ao passo que esse é responsável pela organização do trabalho coletivo da enfermagem, de modo que o cuidado possa ser desenvolvido. Entende-se que a proatividade desse profissional pode acarretar repercussões importantes em seu fazer ${ }^{(4)}$. Diante do cenário prático, salienta-se a importância do gerenciamento do cuidado como foco das ações profissionais, uma vez que os processos administrativos devem ser usados como tecnologias para sua efetivação, por intermédio de ações diretas com os usuários ou por meio da delegação e da articulação com os demais profissionais da equipe de saúde ${ }^{(5)}$.

A assistência à saúde no âmbito do Sistema Único de Saúde (SUS), mesmo com os avanços em prol da consolidação da Estratégia de Saúde da Família (ESF), como modelo de reorientação da atenção básica, possui lacunas com influência do modelo tradicional, caracterizando-se por atividades fragmentadas, hierarquizadas, individualistas e com visão positivista do processo saúde-doença ${ }^{(6)}$.

Cabe mencionar que desde a criação do SUS, houve avanços na sua implementação. O supracitado sistema aumentou consideravelmente o acesso aos cuidados de saúde para grande parcela da população, atingindo a cobertura universal para a vacinação e a assistência pré- natal; ampliou a conscientização da população a respeito do direito à saúde vinculado à cidadania; promoveu o desenvolvimento de recursos humanos e da tecnologia em saúde. Contudo, o SUS consiste em um sistema de saúde que luta de forma contínua, por intermédio de sua sustentabilidade política, econômica, científica e tecnológica, para garantir a cobertura universal e equitativa ${ }^{(7)}$.

Por este motivo, carece de profissionais engajados em busca do desenvolvimento de ações de saúde. Dentre as ações gerencias do enfermeiro no contexto da ESF, destaca-se: participação na elaboração, na coordenação e na articulação do planejamento da Unidade Básica de Saúde (UBS); promoção da integração e do bom relacionamento das equipes de saúde sob sua responsabilidade; investimento nas relações interpessoais (valorizando o desempenho dos profissionais); identificação dos recursos físicos e do perfil demográfico e epidemiológico da população adscrita ${ }^{(6-8)}$.

Destaca-se que o estudo é relevante, tendo em vista que a literatura aponta que a maior parte das investigações referentes ao gerenciamento do cuidado de enfermagem está relacionada ao cuidado hospitalar ${ }^{(6)}$. Além do mais, há limitações das atividades realizadas pelos enfermeiros nas ESF, dentre elas, a falta de autonomia em muitos municípios, por não haver a implementação de protocolos que respaldem suas atividades, o que consequentemente, implica no desempenho de funções, interferindo diretamente no gerenciamento do cuidado ${ }^{(9)}$.

Baseado no exposto, objetivou-se conhecer o entendimento dos enfermeiros atuantes na Estratégia de Saúde da Família acerca do conceito, das dificuldades e as estratégias utilizadas para desenvolver o gerenciamento do cuidado. 


\section{MÉTODOS}

Pesquisa qualitativa, descritiva e exploratória, realizada na rede básica de saúde de Pelotas/RS/Brasil. O município possui 50 UBS, em que 37 possuem equipes com ESF, correspondendo no total, 66 equipes de ESF. Ressalta-se que dentro desses valores, 26 UBS com equipes de ESF estão localizadas na zona urbana.

Participaram do estudo 20 enfermeiros. Adotaram-se os seguintes critérios de inclusão: enfermeiros que atuavam em UBS com ESF na área urbana e estavam na mesma equipe há pelo menos seis meses. Os critérios de exclusão do estudo foram: enfermeiros não encontrados no serviço em mais de duas visitas agendadas ou afastados de suas atividades (licença saúde/maternidade).

O número de participantes foi delimitado pelo critério de saturação, ou seja, quando as informações, explicações e sentidos atribuídos pelos participantes começaram a ter regularidade de apresentação. Já as entrevistas foram agendadas previamente por contato telefônico, sendo selecionado, por meio de sorteio, um enfermeiro de cada UBS contatada quando havia mais de uma equipe de saúde da família.

A coleta de dados ocorreu em setembro de 2015 por meio de entrevista semiestruturada, utilizando um roteiro para identificar o perfil dos participantes e questões abertas, a fim de investigar o conhecimento, a aplicação e a opinião dos enfermeiros sobre o tema investigado. Para preservar o anonimato, os depoimentos foram identificados com a letra E e o número ordinal correspondente à sequência das entrevistas realizadas (E1, E2 ..., E20). Os participantes foram questionados quanto ao entendimento e como realiza o gerenciamento na prática, bem como as dificuldades, facilidades e estratégias para implementá-lo na ESF.

Utilizou-se análise temática, estruturando e organizando o conteúdo em dois momentos. O primeiro refere-se à fase exploratória da investigação, constituindo o marco-teórico fundamental para a análise. 0 segundo é o interpretativo, no qual se utilizou dos relatos dos informantes para dar sentido, lógica e projeção, buscando compreensão e agregação das respostas ${ }^{(10)}$. Primeiramente, realizou-se a ordenação dos dados que englobaram a transcrição do material obtido por meio das entrevistas e releitura do material. Após, iniciou-se a classificação dos dados, por meio da leitura horizontal e exaustiva dos textos, permitindo apreender as estruturas de relevância e as ideias centrais. Na leitura transversal, foram separados os dados por unidades de sentido. No processo classificatório, identificaram-se as categorias, juntando as partes semelhantes, buscando perceber as conexões entre elas, e guardando-as em códigos. Na análise final, os dados foram confrontados com a literatura.

A partir da análise, emergiram três categorias: O entendimento dos enfermeiros das ESF sobre gerenciamento do cuidado de enfermagem; Dificuldades enfrentadas durante a implementação do gerenciamento do cuidado de enfermagem; e Estratégias para melhorar o gerenciamento do cuidado.

Salienta-se que em relação aos princípios éticos, consideraram-se em todas as etapas da pesquisa, as determinações da Resolução 466/12 do Conselho Nacional de Saúde sobre pesquisa com Seres Humanos. 0 projeto possui aprovação do Comitê de Ética em Pesquisa da Faculdade de Enfermagem da Universidade Federal de Pelotas, sob protocolo de número 1.208.951. 


\section{RESULTADOS E DISCUSSÃO}

Quanto ao perfil dos 20 enfermeiros participantes do estudo, a idade variou entre 29 e 58 anos e dois eram do sexo masculino. 0 tempo de formação foi de sete a 28 anos e somente um não era egresso de universidade pública. Nenhum deles realizou curso de auxiliar ou de técnico em enfermagem. Apenas um não tinha pós-graduação; 18 possuíam especialização, e desses, 14 na área de saúde pública ou em saúde da família; dois concluíram o mestrado. O tempo de trabalho na equipe de ESF compreendeu entre oito meses a 15 anos, sendo que nove ocupavam o cargo de coordenadores da equipe multiprofissional. Em relação ao vínculo empregatício, todos eram servidores públicos, com carga horária de trabalho de 40 horas semanais, porém, seis possuíam outro vínculo empregatício, aspecto que pode refletir diretamente na assistência, visto a sobrecarga de trabalho semanal.

\section{O entendimento dos enfermeiros das ESF sobre gerenciamento do cuidado de enfermagem}

No intuito de conhecer o entendimento sobre o gerenciamento do cuidado de enfermagem na visão dos enfermeiros atuantes em ESF, houve a necessidade de questioná-los quanto ao conceito que eles traziam sobre esse tema.

É tudo o que tu faz na unidade para o bom andamento do serviço, desde a organização do atendimento, a forma como tu vais fazer diariamente, a forma como tu planejas os programas, quando tu vais criar vínculos com os pacientes no retorno, nas visitas domiciliares (E15).

Envolve início, meio e fim do atendimento ao usuário, desde escuta, percepção, orientação, planejamento da orientação para o cuidado dos pacientes (E5).

É ter ações programáticas que possibilitam poder cuidar de uma comunidade no geral, que na Estratégia se cuida de toda a família. Deixar de ter aquele olhar só no problema e passa a ver o problema familiar (E10).

É interessante o fato de que esses profissionais entendem que assistência e gerenciamento se articulam. Eles percebem que toda a estrutura e a organização da unidade interferem diretamente no cuidado prestado à população. Na medida em que eles conseguem organizar o seu processo de trabalho, sentem a repercussão no bom atendimento.

O enfermeiro vem redescobrindo o seu papel na Atenção Primária à Saúde (APS), ao longo de sua vivência, criando e recriando o fazer em enfermagem na saúde pública, tanto no que diz respeito no cuidado como na promoção da saúde ${ }^{(11)}$. Ao desempenhar sua função social de cuidador, ele convive com as tensões próprias da produção dos atos de saúde, ou seja, a produção de procedimentos versus a produção de cuidado, o que vai ao encontro dos resultados obtidos neste estudo ${ }^{(12)}$. Além disso, busca-se alcançar a ruptura de pressupostos tradicionais do gerenciamento em enfermagem nos serviços de saúde e compreende-se que a melhor maneira do enfermeiro estimular sua equipe é considerar cada integrante como fundamental no processo, investindo no potencial individual e coletivo, com vistas atingir as metas estabelecidas $^{(13)}$.

Por meio desses resultados, foi possível entender que gerenciar o cuidado na ESF é articular atividades assistenciais e gerenciais do enfermeiro para se ter um planejamento de cuidado a cada usuário dos serviços, 
norteado pelas políticas preconizadas pelo Ministério da Saúde.

\section{Dificuldades enfrentadas durante a implementação do gerenciamento do cuidado de enfermagem}

Dentre as dificuldades relatadas pelos participantes no gerenciamento do cuidado de enfermagem, a falta de estrutura física e de materiais foi ressaltada pelos entrevistados.

As maiores dificuldades são a falta de recursos, a falta de estrutura física (E6).

São atender as urgências, não tem medicamentos para urgência, não tem materiais, não tem ambu [máscara de oxigênio], oxigênio, rede de oxigênio. Preciso encaminhar para o hospital de referência, chamo SAMU (E4).

A insatisfação com a estrutura física foi relatada pela maioria dos participantes, exceto uma unidade que recentemente havia recebido incentivo financeiro de uma organização privada, pouco envolvendo os recursos da gestão municipal.

Frente ao exposto, salienta-se que a expansão da cobertura e do financiamento público é imprescindível para oferecer atenção similar a todos os pacientes do SUS, e promover a efetiva inserção do setor privado numa rede regionalizada e racionalizada de serviços de saúde. As complexas dimensões da combinação entre público-privado na saúde estão ainda por ser adequadamente enfrentadas ${ }^{(14)}$. Diante disso, destaca-se que o trabalho do enfermeiro envolve a gestão do cuidado, além de outras questões que, muitas vezes, saem de sua alçada, mas que necessitam de seu olhar crítico e participativo.

Neste estudo, as unidades que tiveram a estrutura física recém-reformada foram consideradas ruins, pois ficaram com muitas pendências. Ficou visível também, o quanto essa questão interfere na motivação dos profissionais, já que vários carecem de uma sala para realizar as consultas de enfermagem e grande parte das estruturas sofre com a proliferação de mofo, devido à característica climática da cidade a qual predispõe à umidade em níveis elevados. Ainda cabe salientar, que as condições estruturais e organizacionais impactam diretamente na oferta da consulta de enfermagem, atividade privativa do enfermeiro, a qual pode não ser ofertada por falta de espaço físico ou porque a organização do processo de trabalho na ESF pode também ser manter sendo realizada na lógica biomédica.

Nessa perspectiva, estudo realizado no Município de Ribeirão Preto/SP/Brasil reforça que algumas condições estruturais e organizacionais das unidades de saúde, são assinaladas como dificuldades na rotina da prática clínica dos enfermeiros na atenção básica. As condições arquitetônicas e organizacionais de algumas unidades de saúde não facilitam à oferta desse atendimento, pois, historicamente, ocorre a atenção em saúde centrada no modelo biomédico ${ }^{(12)}$.

Embora em número pequeno, foi citada como dificuldade, a resistência que se tem em trabalhar em equipe multiprofissional, principalmente em relação aos médicos. Esse impasse foi observado nas equipes que contam com profissionais que já trabalhavam na unidade quando ainda a ESF era tradicional, o qual havia uma proposta de trabalho diferente.

É a dificuldade de trabalhar com profissionais, essa resistência que eles têm (E8). 
Outro estudo realizado em um município do Estado do Paraná/Brasil também corrobora com esse depoimento, pois os seus entrevistados citaram como dificuldades entre os integrantes da equipe multiprofissional a resistência, principalmente, relacionada às mudanças, bem como a falta de tolerância e o déficit na comunicação entre a equipe ${ }^{(15)}$. A questão da resistência encontrada pode estar relacionada à existência de uma relação de poder entre os envolvidos na equipe de trabalho, pois segundo Michel Foucault ${ }^{(16)}$, quando existe poder, existe resistência.

A autonomia foi vista como uma facilidade por alguns enfermeiros e como dificuldade para outros. Essa divergência de opiniões pode estar relacionada à forma como as equipes organizam o seu processo de trabalho, de modo que não construam uma hierarquização entre os profissionais, fazendo com que a equipe perceba a importância de todos e o direito de cada um ter espaço físico adequado para os atendimentos.

Eu sinto que nos falta um pouco de autonomia, um pouco de espaço físico para que a gente tenha uma liberdade maior com os nossos pacientes (E9).

Indo ao encontro do que foi mencionado, almeja-se que cada profissional da equipe tivesse e procurasse autonomia para resolver ou buscar recursos para os problemas do seu território de atuação. Contudo, essa autonomia muitas vezes não é buscada, e quando é, esbarra em processos burocráticos como filas, baixa resolução de problemas por parte de outros profissionais, desarticulação da rede quando há necessidade de encaminhamento para outros setores, entre outros ${ }^{(17)}$.

Em um estudo realizado com equipes de saúde da família do modelo de atenção tradicional, no Sul do Brasil, demonstrou importantes fragilidades encontradas pelos profissionais. Tais dimensões que elas atingem na atenção básica são de ordem estrutural, política e cultural, ressaltando o quanto essas dificultam a implantação dos princípios do SUS, influenciando negativamente na rotina de trabalho dos profissionais de saúde ${ }^{(18)}$.

Somando-se a isso, também está a influência política, a qual pode ser considerada uma das fragilidades das dimensões da atenção básica. Situação complexa de ser resolvida, mas que se fosse possível rever algumas situações, traria mudanças significativas diante da realidade das UBS, pois, atualmente se tem um grande impasse voltado a essa questão, a inclusão dos gerentes distritais, um novo cargo instituído pela administração municipal.

É o gerente distrital quem decide e não eu. Não são concursados, são cargos políticos, não tenho nada contra as pessoas. A politicagem na área da saúde faz as coisas não andarem. Devem ser concursados e ter prática na área da saúde (E14).

O envolvimento na política partidária interfere diretamente no planejamento do cuidado em saúde. Há a tradição política no país e, o dissociar as políticas públicas sociais da ação política partidária ainda é complexo, pois há influências que levam prejuízos na condução dessas ações sociais, como são caracterizadas na $\mathrm{ESF}^{(19)}$.

Evidencia-se o quanto essas dificuldades interferem no gerenciamento do cuidado desses 
profissionais. Mesmo sabendo que o comprometimento com o trabalho e a vontade de querer fazer o melhor sejam ferramentas fundamentais nesse processo, há certos recursos imprescindíveis, principalmente o apoio da gestão e o trabalho junto as ESF.

\section{Estratégias para melhorar o gerenciamento do cuidado}

Entre as estratégias para melhorar o gerenciamento do cuidado, observou-se a necessidade de maior aproximação da gestão municipal junto às unidades, a fim de estarem mais próximos da realidade para um melhor planejamento e apoio às suas necessidades.

Aproximação do pessoal da Coordenação com o pessoal que trabalha no Posto (E1).

Planejamento em primeiro lugar. Temos que ver qual é a realidade da comunidade que atendemos (E9).

$O$ apoio da gestão. Oportunidade de tu poderes discutir as coisas e organizar melhor e poder trabalhar melhor em cima daquilo que tu acreditas e que é o certo. Não em cima de politicagem (E15).

Na verdade, acredito que se os gerentes distritais se ocupassem em ver quais são as necessidades de cada unidade, tanto no que diz respeito aos insumos, materiais, tudo o que for viável para melhorar, porque têm coisas que são de boa vontade (E17).

Conforme os depoimentos, ao assumir determinado cargo na gestão, é imprescindível conhecer as atribuições e a realidade do seu trabalho. Ao comparar com essa afirmativa, destaca-se um estudo realizado com enfermeiros da 10a Regional de Saúde do Estado do Paraná/Brasil que acredita ocorrer mudança na forma de pensar dos gestores e se estes começarem a entender os propósitos e a realidade da ESF, em cada localidade, haverá mudança na destinação dos recursos, melhorando as condições de trabalho de toda a equipe $^{(20)}$.

Outra estratégia com vistas a facilitar o gerenciamento do cuidado nas ESF, citada pelos participantes, foi a padronização do atendimento ofertado nas unidades, por intermédio da criação de protocolos, juntamente com a manutenção da educação continuada como forma de sustentar as suas práticas.

Da parte da gestão, se a gente tivesse mais capacitações envolvendo este tema, se fizesse mais a padronização. Acho que a criação de protocolos (E2).

Educação continuada e a sistematização por protocolos (E7).

Acredito que mais reuniões entre equipes de Estratégia para discutir sobre o tema [gerenciamento do cuidado], porque assim, cada um vai ver a dificuldade do outro e o que o outro conseguiu fazer de positivo e é algo que na cidade não se tem. Mais coisas pessoais, do profissional ir buscar também (E13).

Ao analisar esses depoimentos, identificou-se carência nas atividades voltadas à educação continuada, pois de acordo com as novas políticas de saúde, mudanças são necessárias para a formação dos profissionais de saúde, iniciando-se desde a graduação e mantendo-se após a inserção no mercado de trabalho ${ }^{(21)}$. Assim, os entrevistados demonstraram quais demandas de suas rotinas necessitam de atualização. Contudo, ficou visível que além da contribuição da gestão, o profissional também deve fazer a sua parte e ir em busca do conhecimento na tentativa de suprir parte de suas necessidades. Ressalta-se ainda, as cobranças que possuem frente ao seu trabalho, mas que ao mesmo tempo, a gestão tem dificuldades em entender a 
realidade laboral das unidades.

A falta de protocolos de enfermagem no Município de Pelotas/RS/Brasil faz com que muitas atividades não aconteçam devido à falta de respaldo legal. Embora se saiba que alguns manuais já foram estabelecidos pelo Ministério da Saúde para a execução de atividades pelo profissional enfermeiro ${ }^{(9)}$. Assim, o acolhimento utilizado na ESF seria relevante por auxiliá-lo a organizar os seus atendimentos e por ser um bom instrumento para a implementação de protocolos e do processo de enfermagem no cuidado aos usuários que necessitam de maior atenção devido a sua vulnerabilidade ${ }^{(22)}$.

A melhoria no que se refere aos recursos humanos disponíveis nas equipes das ESF também foi uma das estratégias sugeridas pelos participantes, como forma de garantir o cuidado através da equipe completa, conforme preconizado pelo Ministério da Saúde.

Se a gente tivesse as equipes completas, já daria para organizar, mesmo sem estrutura física (E3). Aumentar os recursos humanos de maneira qualitativa e também fazer com que esta gerência seja feita por pessoas da área da saúde com comprovação da competência e não por cargo político (E14).

Quando alguém entra de férias, eles [gestão] não mandam outro para substituir (E19).

Frente a essa situação, a indisponibilidade de profissionais atuantes em uma equipe de saúde juntamente com a falta de qualificação, tem a tendência de influenciar negativamente na prestação dos cuidados às famílias $^{(20)}$. Nesse contexto, a falta de um dos integrantes da equipe multiprofissional, repercute negativamente na organização das atividades diárias da unidade, sobrecarregando os profissionais que estão atuando e, consequentemente, repercutindo na qualidade da assistência prestada à população.

\section{CONCLUSÃO}

O gerenciamento do cuidado é entendido como uma atividade prioritariamente desenvolvida pelo enfermeiro, com vistas a qualificar a qualidade do cuidado e melhorar as condições de trabalho dos profissionais de saúde.

Quanto às dificuldades enfrentadas pelos enfermeiros para gerenciar o cuidado nas unidades, podese destacar a insatisfação e o descontentamento com relação à gestão municipal. A identificação de tais entraves possibilita o repensar do processo de trabalho gerencial do enfermeiro, bem como permite apontar para a necessidade de novas formas de fazer saúde, transpondo a visão tradicional que ainda deixa resquícios nos serviços de saúde.

Evidenciou-se no estudo a necessidade de padronização das atividades e protocolos, maior aproximação da gestão municipal com os seus serviços e capacitações sobre os programas que são desenvolvidos na unidade. Tais estratégias podem contribuir com o fortalecimento do gerenciamento do cuidado, contudo, demonstram que muitas decisões ultrapassam o poder decisório do enfermeiro, o que reflete na necessidade de gestores públicos sensíveis à implementações de propostas que respaldem e potencializem as ações dos enfermeiros na ESF.

Dentre as limitações do estudo, destaca-se que se utilizou uma única técnica de coleta de dados, a 
entrevista. A associação de outras técnicas como a observação, poderia ter auxiliado a reforçar os dados obtidos e facilitado na compreensão dos relatos, por observar o contexto de trabalho nos quais estão inseridos.

A partir do desenvolvimento deste estudo, buscou-se contribuir para a reflexão sobre o gerenciamento do cuidado dos enfermeiros atuantes em ESF, a fim de melhorar suas práticas e, consequentemente, a qualidade do cuidado prestado à população nos mais diversos territórios.

\section{REFERÊNCIAS}

1. Santos JLG, Pestana AL, Guerrero P, Meirelles BSH, Erdmann AL. Práticas de enfermeiros na gerência do cuidado em enfermagem e saúde: revisão integrativa. Rev Bras Enferm [Internet]. 2013 [acesso em: 17 abr. 2017];66(2):257-63. Disponível em: http://dx.doi.org/10.1590/S0034-71672013000200016.

2. Chaves LDP, Camelo SHH, Laus AM. Mobilizando competências para o gerenciamento do cuidado de enfermagem. Rev. Eletr. Enf. [Internet]. 2011 [acesso em: 17 abr. 2017];13(4):594. Disponível em:

https://doi.org/10.5216/ree.v13i4.13406.

3. Christovam BP, Porto IS, Oliveira DC. Nursing care management in hospital settings: the building of a construct. Rev Esc Enferm USP [Internet]. 2012 [acesso em: 17 abr. 2017];46(3):734-41. Disponível em:

http://dx.doi.org/10.1590/S0080-62342012000300028.

4. Ferreira GE, Dall'Agnol CM, Porto AR. Repercussões da proatividade no gerenciamento do cuidado: Percepções de enfermeiros. Esc. Anna Nery [Internet]. 2016 [acesso em: 17 abr. 2017];20(3):e20160057. Disponível em:

http://dx.doi.org/10.5935/1414-8145.20160057.

5. Santos JLG, Lima MADS. Gerenciamento do cuidado: ações dos enfermeiros em um serviço hospitalar de emergência. Rev Gaucha Enferm [Internet]. 2011 [acesso em: 17 abr. 2017];32(4):695-702. Disponível em: http://dx.doi.org/10.1590/\$1983-14472011000400009.

6. Fernandes MC, Silva LMS. Gerência do cuidado do enfermeiro na estratégia saúde da família: revisão integrativa. Rev Rene [Internet]. 2013 [acesso em: 17 abr. 2017];14(2):438-47. Disponível em:

http://www.periodicos.ufc.br/index.php/rene/article/view/3407.

7. Paim J, Travassos C, Almeida C, Bahia L, Macinko J. The Brazilian health system: history, advances, and challenges. Lancet [Internet]. 2011 [acesso em: 17 abr. 2017];377(9779):1778-97. Disponível em:

http://dx.doi.org/10.1016/s0140-6736(11)60054-8.

8. Kawata LS, Mishima SM, Chirelli MQ, Pereira MJB, Matumoto S, Fortuna CM. Attributes mobilized by nurses in Family Health: reaching performances when developing managerial competence. Rev Esc Enferm USP [Internet]. 2011 [acesso em: 17 abr. 2017];45(2):349-55. Disponível em: http://dx.doi.org/10.1590/\$0080-62342011000200007. 9. Ceolin T, Heck RM, Casarin ST, Ceolin AR. Processo de trabalho dos enfermeiros na Estratégia de Saúde da Família. Enfermería Comunitaria [Internet]. 2009 [acesso em: 17 abr. 2017];5(1). Disponível em: http://www.index-

f.com/comunitaria/v5n1/ec6986r.php.

10. Minayo M. O desafio do conhecimento: pesquisa qualitativa em saúde. 12ª ed. São Paulo: Editora Hucitec, 2010. 11. Oliveira MM, Coimbra VCC, Oliveira EM, Pereira DB, Matins A. O profissional enfermeiro e a atenção primária à saúde. Journal of Nursing and Health [Internet]. 2011 [acesso em: 17 abr. 2017];1(1):184-9. Disponível em: https://periodicos.ufpel.edu.br/ojs2/index.php/enfermagem/article/view/3422.

12. Matumoto S, Fortuna CM, Kawata LS, Mishima SM, Pereira MJB. Nurses' clinical practice in primary care: a process under construction. Rev Lat Am Enfermagem [Internet]. 2011 [acesso em: 17 abr. 2017];19(1):123-30. Disponível em: http://dx.doi.org/10.1590/\$0104-11692011000100017.

13. Oliveira FEL, Fernandes SCA, Oliveira LL, Queiroz JC, Azevedo VRC. A gerência do enfermeiro na Estratégia Saúde da Família. Rev Rene [Internet]. 2012 [acesso em: 17 abr. 2017];13(4):834-44. Disponível em:

http://www.periodicos.ufc.br/index.php/rene/article/view/4043.

14. Victora CG, Barreto ML, Leal MC, Monteiro CA, Schmidt MI, Paim J, et al. Health conditions and health-policy innovations in Brazil: the way forward. Lancet [Internet]. 2011 [acesso em: 17 abr. 2017];377(9782):2042-53. 
Disponível em: http://dx.doi.org/10.1016/S0140-6736(11)60055-X.

15. Navarro ASS, Guimarães RLS, Garanhani ML. Trabalho em equipe: o significado atribuído por profissionais da estratégia de saúde da família. REME [Internet]. 2013 [acesso em: 17 abr. 2017];17(1):61-8. Disponível em:

http://www.dx.doi.org/10.5935/1415-2762.20130006.

16. Foucault M. História da sexualidade I: a vontade de saber. Rio de Janeiro: Edições Graal; 1988.

17. Sena AL, Cavalcanti RP, Pereira IL, Leite SRR. Intersetorialidade e ESF: limites e possibilidades no território de uma unidade integrada de Saúde da Família. Revista Brasileira de Ciências da Saúde [Internet]. 2012 [acesso em: 17 abr. 2017];16(3):337-42. Disponível em: http://dx.doi.org/10.4034/RBCS.2012.16.03.09.

18. Trindade LL, Pires DEP. Implications of primary health care models in workloads of health professionals. Texto contexto - enferm. [Internet]. 2013 [acesso em: 17 abr. 2017];22(1):36-42. Disponível em: http://dx.doi.org/10.1590/S0104-07072013000100005.

19. Medeiros CRG, Junqueira ÁGW, Schwingel G, Carreno I, Jungles LAP, Saldanha OMFL. A rotatividade de enfermeiros e médicos: um impasse na implementação da Estratégia de Saúde da Família. Cien Saude Colet [Internet]. 2010 [acesso em: 17 abr. 2017];15 supl. 1:1521-31. Disponível em: http://dx.doi.org/10.1590/S141381232010000700064.

20. Roecker S, Budó MLD, Marcon SS. The educational work of nurses in the Family Health Strategy: difficulties and perspectives on change. Rev Esc Enferm USP [Internet]. 2012 [acesso em: 17 abr. 2017];46(3):641-9. Disponível em: http://dx.doi.org/10.1590/S0080-62342012000300016.

21. Varela DSS, Carvalho MMB, Barbosa MUF, Silva IZF, Gadelha RRM, Machado MFAS. Diretrizes Curriculares Nacionais e a Formação de Profissionais para o SUS. Revista Brasileira de Educação e Saúde [Internet]. 2016 [acesso em: 17 abr. 2017];6(3):39-43. Disponível em: http://www.gvaa.com.br/revista/index.php/REBES/article/view/3928. 22. Costa RHS, Couto CRO, Silva RAR. Prática clínica do enfermeiro na Estratégia de Saúde da Família. Saúde (Santa Maria) [Internet].; 2015 [acesso em: 17 abr. 2017];41(2):9-18. Disponível em:

http://dx.doi.org/10.5902/2236583410841. 